\title{
A new technique for mapping of total electron content using GPS network in Japan
}

\author{
Y. Otsuka ${ }^{1}$, T. Ogawa ${ }^{1}$, A. Saito ${ }^{2,3}$, T. Tsugawa ${ }^{4}$, S. Fukao ${ }^{5}$, and S. Miyazaki ${ }^{6}$ \\ ${ }^{1}$ Solar-Terrestrial Environment Laboratory, Nagoya University \\ ${ }^{2}$ School of Electrical and Computer Engineering, Cornell University \\ ${ }^{3}$ On leave from Department of Geophysics, Graduate School of Science, Kyoto University \\ ${ }^{4}$ Department of Geophysics, Graduate School of Science, Kyoto University \\ ${ }^{5}$ Radio Science Center for Space and Atmosphere, Kyoto University \\ ${ }^{6}$ Geographical Survey Institute
}

(Received October 31, 2000; Revised December 7, 2001; Accepted December 7, 2001)

\begin{abstract}
The dual frequency radio signals of the Global Positioning System (GPS) allow measurements of the total number of electrons, called total electron content (TEC), along a ray path from GPS satellite to receiver. We have developed a new technique to construct two-dimensional maps of absolute TEC over Japan by using GPS data from more than 1000 GPS receivers. A least squares fitting procedure is used to remove instrumental biases inherent in the GPS satellite and receiver. Two-dimensional maps of absolute vertical TEC are derived with time resolution of 30 seconds and spatial resolution of $0.15^{\circ} \times 0.15^{\circ}$ in latitude and longitude. Our method is validated in two ways. First, TECs along ray paths from the GPS satellites are simulated using a model for electron contents based on the IRI-95 model. It is found that TEC from our method is underestimated by less than 3 TECU. Then, estimated vertical GPS TEC is compared with ionospheric TEC that is calculated from simultaneous electron density profile obtained with the MU radar. Diurnal and day-to-day variation of the GPS TEC follows the TEC behavior derived from MU radar observation but the GPS TEC is 2 TECU larger than the MU radar TEC on average. This difference can be attributed to the plasmaspheric electron content along the GPS ray path. This method is also applied to GPS data during a magnetic storm of September 25, 1998. An intense TEC enhancement, probably caused by a northward expansion of the equatorial anomaly, was observed in the southern part of Japan in the evening during the main phase of the storm.
\end{abstract}

\section{Introduction}

The dual frequency radio signals of the Global Positioning System (GPS) at an altitude of 20,200 km allow the measurement of the total number of free electrons, called total electron content (TEC), along ray path from GPS satellite to receiver. This TEC, however, usually includes instrumental biases inherent in the GPS receivers and transmitters. These biases must be removed to obtain absolute values of TEC. Several techniques to calculate absolute TEC using the Kalman filter have been proposed. Sardón et al. (1994) estimated absolute TEC assuming that vertical TEC distribution is spatially linear around the zenith of the receiver. Bishop et al. (1996) developed a self-calibration of pseudorange errors (SCORE) technique which was validated by independent measurements (Bishop et al., 1997) and modeling (Lunt et al., 1999a). These techniques can be applied for data from a single GPS receiver. Mannucci et al. (1998) produced global maps of vertical TEC by interpolating TEC of more than 60 worldwide GPS stations in order to study

*Present address: 3-13 Honohara, Toyokawa, Aichi 442-8507, Japan.

Copy right (c) The Society of Geomagnetism and Earth, Planetary and Space Sciences (SGEPSS); The Seismological Society of Japan; The Volcanological Society of Japan; The Geodetic Society of Japan; The Japanese Society for Planetary Sciences. a global distribution of TEC under magnetically disturbed conditions. Ho et al. (1996), using such global TEC maps, found significant TEC increases in the winter hemisphere during a magnetic storm. Buonsanto et al. (1999) compared global TEC maps with incoherent scatter radar data from Sondrestrom, Millstone Hill, Arecibo, and Jicamarca to study the May 1-5, 1995 storm event.

The Geographical Survey Institute (GSI) of Japan has launched its nationwide GPS array project in April 1994 and has installed more than 1000 receivers in Japan, called GPS Earth Observation Network (GEONET) (Miyazaki et al., 1997). The average distance between two receivers is approximately $25 \mathrm{~km}$. One GPS receiver measures signals simultaneously from typically $6-7$ satellites. GPS data are sampled every 30 seconds. These conditions allow the retrieval of TEC maps over Japan with high spatial resolution and a time resolution of 30 seconds. The above-mentioned techniques to obtain absolute TEC are not applicable to GEONET data gathered from a large number of receivers, because it takes much computation time to use the Kalman filter for each update epoch.

In the present study, we develop a new simple and robust technique to derive two-dimensional map of absolute TEC over Japan by using the GEONET data. This method provides absolute TEC as a function of time and location. 
Simulated observations of TEC along ray paths from GPS satellites are used to validate this technique. The results are compared with MU radar observations under geomagnetically quiet conditions.

\section{Estimation of Absolute TEC and Instrumental Biases}

GPS observations provide both carrier phase delays $L$ and pseudoranges $P$ of the dual frequencies $\left(f_{1}=1575.42 \mathrm{MHz}\right.$ and $f_{2}=1227.60 \mathrm{MHz}$ ). Phase and group delays of radio waves in the ionosphere, $d_{p}$ and $d_{g}$ (in meters), respectively, are proportional to total electron content integrated along propagation path, $T$ (in electrons per square meter)

$$
-d_{p}=d_{g}=\frac{40.3 T}{f^{2}}
$$

where $f$ is the radio wave frequency in Hertz (e.g., Davies, 1990). Since the neutral gas influence on the refractive index is non-dispersive and does not depend on frequency, TEC can be derived by comparing the phase delays of $L_{1}$ and $L_{2}$ signal. $1 \mathrm{~ns}$ of differential time delay corresponds to 2.852 TEC units (TECU) where 1 TECU $=1 \times 10^{16}$ electrons $/ \mathrm{m}^{2}$. The difference of the carrier phase delays $L_{I}=L_{1}-L_{2}$ tracks very precisely TEC. However, sudden $L_{I}$ changes (cycle slip) sometimes occur when a GPS receiver losses lock on the GPS signals. The cycle slips are detected as discontinuity in $L_{I}$ and corrected using an algorithm developed by Belwitt (1990). Although TEC derived from the phase data provides relative change of TEC, a level of TEC is unknown because of unknown initialization constant in phase data. Therefore, the level of TEC is adjusted to that of TEC derived from the corresponding pseudorange difference $\left(P_{2}-P_{1}\right)$ for each satellite-receiver pair. The level is determined for each set of connected TECs. TEC data separated over more than 15 minutes in time are not connected. The pseudorange delays still contain delays inherent in satellite and receiver hardwares. To get absolute TEC, these biases must be removed.

As described above, absolute TEC, $I^{i}(t)$, measured by satellite- $i$ at epoch time $t$ is determined from the phase and pseudorange data. $I^{i}(t)$ consists of both the slant TEC, $T^{i}(t)$, along a satellite-receiver ray path and the instrumental bias $B^{i}$.

$$
I^{i}(t)=T^{i}(t)+B^{i}
$$

The instrumental biases are considered to be constant over several days (Sardón et al., 1994; Sardón and Zarraoa, 1997). We assume that the ionosphere is a thin shell. The thin-shell model assumption is commonly used in GPS TEC studies (e.g., Bishop et al., 1997; Sardón et al., 1994). From this assumption, the slant TEC, $T^{i}(t)$, at a give point in the ionospheric shell is related to the equivalent vertical TEC, $V^{i}(t)$, at that point by

$$
T^{i}(t)=S\left(\epsilon^{i}(t)\right) V^{i}(t)
$$

where $S\left(\epsilon^{i}(t)\right)$ is the slant factor and $\epsilon^{i}(t)$ is the elevation angle of the GPS satellite. Thus $I^{i}(t)$ is written as follows:

$$
I^{i}(t)=S\left(\epsilon^{i}(t)\right) V^{i}(t)+B^{i}
$$

The ionization distribution is approximated by a slab of constant-density with the lower and upper boundary at altitudes of 300 and $550 \mathrm{~km}$, respectively. Then, the slant factor $S$ is defined as $S=\tau_{1} / \tau_{0}$, where $\tau_{1}$ is the length of the ray path between 300 and $550 \mathrm{~km}$ altitudes and $\tau_{0}$ is the ionosphere thickness of $250 \mathrm{~km}$ for zenith path. However, actual electron density distribution is not so simple as this assumption and extends toward higher (the plasmasphere) and lower (the $E$ region) altitudes. The assumption, therefore, causes some errors in the estimation of vertical TECs. The error increases with increasing slant factor $S$ at lower elevation angle. For example, $S$ is 1.73 at elevation angle of $30^{\circ}$. For this reason, data with elevation angles less than $30^{\circ}$ are excluded in our method.

The procedure to construct a two-dimensional map of absolute vertical TEC with high time and spatial resolution consists of the following three steps. First, hourly averages of TEC are estimated by applying a weighted least squares fitting procedure to the GPS data from a single receiver. Next, the instrumental biases are determined by using the hourly TEC average that is obtained in the first step. Finally, the biases are removed from the measured TEC to derive absolute TECs. Two-dimensional maps of absolute vertical TEC with a time resolution of 30 seconds and a spatial resolution of $0.15^{\circ} \times 0.15^{\circ}$ in latitude and longitude are constructed through these steps. We describe the detailed procedures in these steps below.

(1) Estimation of hourly TEC average: Equation (4) is divided by $S\left(\epsilon^{i}\right)$ and averaged over one hour to estimate the hourly TEC average. Here, we assume that the hourly average of vertical TEC is uniform within an area covered by a receiver; this area approximately corresponds to a surrounding of $1000 \mathrm{~km}$. This assumption means that the hourly vertical TECs obtained from different satellite links by one receiver are the same and can be averaged to a value of vertical TEC, $\overline{V_{k}}$, for each time $k$ where $k$ denotes the index of time sequence of hourly TEC average. Thus, the following equation is derived from Eq. (4).

$$
\overline{I_{k} / S\left(\epsilon_{k}^{i}\right)}=\overline{V_{k}}+\overline{1 / S\left(\epsilon_{k}^{i}\right)} B^{i}
$$

for $k=1,2, \ldots, N_{t}$ and $i=1,2, \ldots, N_{s}$ where $N_{t}$ is the number of hourly TEC average, and $N_{s}$ is the number of satellites which are observed by one receiver. Here, variables with overline denote averaged values.

The number of equations is usually larger than that of the unknowns $\left(\overline{V_{k}}\right.$ with $k=1,2, \ldots, N_{t}$ and $B^{i}$ with $i=$ $\left.1,2, \ldots, N_{s}\right)$. The unknowns are determined through a least squares fitting procedure minimizing the following residual:

$$
E=\sum_{i}^{N_{s}} \sum_{k}^{N_{t}} W_{k}^{i}\left[\overline{I_{k} / S\left(\epsilon_{k}^{i}\right)}-\left(\overline{V_{k}}+\overline{1 / S\left(\epsilon_{k}^{i}\right)} B^{i}\right)\right]^{2}
$$

where $W_{k}^{i}$ is the weighting function. Taking the partial derivatives of $E$ with respect to $\overline{V_{k}}$ and $B^{i}$ and setting them equal to zero yields equations which can be solved for the desired parameters $\left(\overline{V_{k}}\right.$ and $\left.B^{i}\right)$. To reduce the estimation errors of hourly TEC average caused by the assumptions of both the thin-shell model and the spatial uniformity of the hourly TEC average, we select $W_{k}^{i}$ as an inverse of the slant 
factor:

$$
W_{k}^{i}=\overline{1 / S\left(\epsilon_{k}^{i}\right)}
$$

$W_{k}^{i}$ becomes smaller with decreasing elevation angle. Since the GPS satellites appear over a fixed receiver every about 24 hours, our procedure requires continuous data over more than 24 hours at the receiver. In the present study, we use a data set consisting of 24-hour data $\left(N_{t}=24\right)$. This procedure is applied to the GPS data from the GEONET consisting of more than 1000 receivers. We derive more than 1000 data sets of the hourly TEC averages $\left(\overline{V_{k}}, k=1,2, \ldots, N_{t}\right)$ and the instrumental biases $\left(B^{i}, i=1,2, \ldots, N_{s}\right)$.

(2) Estimation of instrumental biases: Hourly TEC averages are mapped on the ionospheric shell which is assumed to be located at $400 \mathrm{~km}$ altitude. Since the receivers are closely distributed (average distance between two receivers is about $25 \mathrm{~km}$ ), areas where different receivers provide TEC estimates overlap. These overlapped TECs are smoothed to obtain the hourly TEC averages within a location of $1^{\circ} \times 1^{\circ}$ in latitude and longitude. This smoothing suppresses isolated outliers and reduces estimation errors of the hourly TEC average derived from the least squares fitting procedure. Substituting the hourly average and spatially smoothed TECs into Eq. (5) determines the instrumental biases $B^{i}$. This new $B^{i}$ is more accurate due to spatially smoothing of the hourly TEC averages than the previous $B^{i}$ derived by the least square fitting procedure in step (1).

(3) Mapping of absolute TEC: Substituting $B_{i}$ into Eq. (4) gives vertical TEC, $V^{i}(t)$, at a sampling interval of $t$ (30 seconds). Absolute vertical TEC $V^{i}(t)$ are mapped on the ionospheric shell at the $400 \mathrm{~km}$ altitude with a pixel of $0.15^{\circ} \times 0.15^{\circ}$ in latitude and longitude. $V^{i}(t)$ within one pixel are averaged. Thus, two-dimensional maps of vertical TEC with a spatial resolution of $0.15^{\circ} \times 0.15^{\circ}$ in latitude and longitude are derived every 30 seconds.

This technique to derive absolute TEC uses data from a large number of closely-distributed GPS receivers and does not need interpolation of TEC in space and time. This technique is also applicable to data from a single receiver. In this case, however, two-dimensional maps of TEC are not obtained because of scarcity of TEC distribution. Only a dense distribution of GPS receivers, such as GEONET, provides two-dimensional map of absolute TEC as a function of location and time.

\section{Validation of the Method \\ 3.1 Model simulation}

We have examined the expected accuracy of this technique using computer simulations. To simulate actual measurements of TEC observed at ground GPS stations in Japan, we use a model for electron contents based on the International Reference Ionosphere (IRI-95) model (Bilitza, 1990) and the results of the Sheffield University Plasmasphere and Ionosphere Model (SUPIM) (Balan et al., 2002). The electron contents in the altitude range of 100-1,000 km are taken from the IRI-95 model. The electron content from the IRI95 varies with altitude, local time, latitude and longitude.

Electron contents above 1,000 km altitude are extrapolated to match the results reported by Balan et al. (2002).

(a)

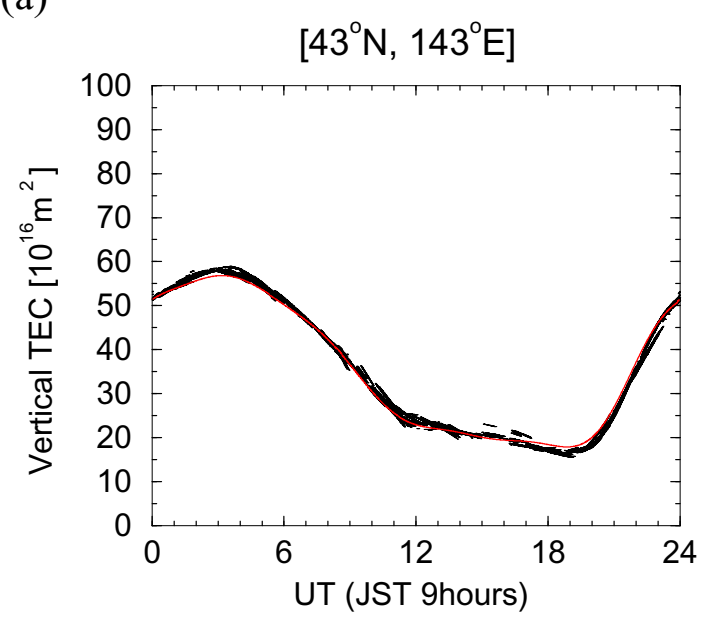

(b)

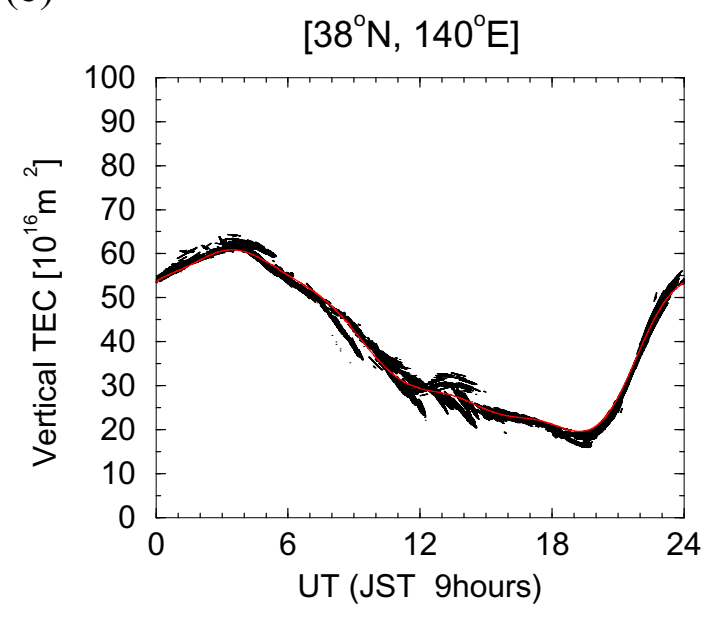

(c)

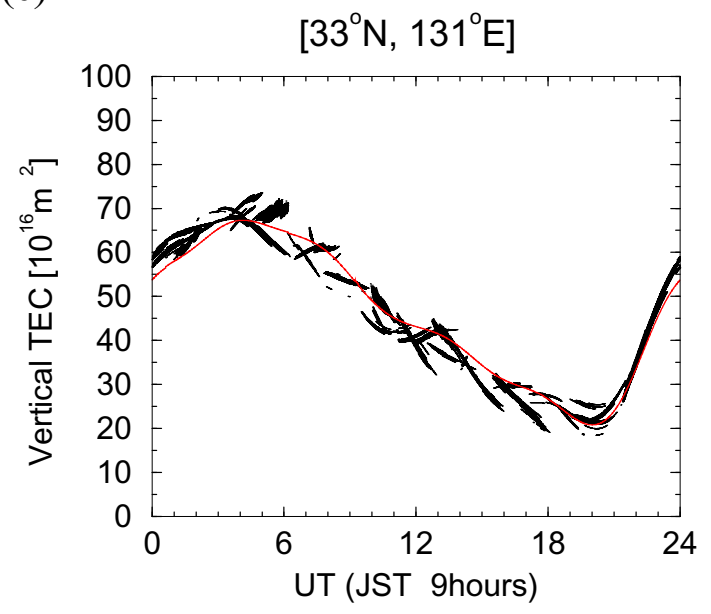

Fig. 1. Examples of the accuracy of the technique estimating absolute vertical TEC over three locations in Japan for equinox at solar maximum. Latitude and longitude of the location are labeled on the top of each figure. The red curves show the vertical TEC obtained by integration of the electron density between $100 \mathrm{~km}$ and the GPS altitude of $20,200 \mathrm{~km}$, which are input of the estimation procedure of absolute TEC. The dotted points show TECs within an area of $1^{\circ} \times 1^{\circ}$ in longitude and latitude derived from the estimation procedure. 
Table 1. Accuracy of the estimation procedure.

\begin{tabular}{lccr}
\hline & $\begin{array}{c}\text { Location } 1 \\
{\left[43^{\circ} \mathrm{N}, 143^{\circ} \mathrm{E}\right]}\end{array}$ & $\begin{array}{c}\text { Location } 2 \\
{\left[38^{\circ} \mathrm{N}, 140^{\circ} \mathrm{E}\right]}\end{array}$ & \multicolumn{1}{c}{$\begin{array}{c}\text { Location } 3 \\
{\left[33^{\circ} \mathrm{N}, 131^{\circ} \mathrm{E}\right]}\end{array}$} \\
\hline Solar maximum equinox & $-2.0 \pm 0.1 \mathrm{TECU}$ & $-2.5 \pm 1.4 \mathrm{TECU}$ & $-1.9 \pm 2.7 \mathrm{TECU}$ \\
Solar maximum winter & $-0.4 \pm 0.9 \mathrm{TECU}$ & $0.0 \pm 1.6 \mathrm{TECU}$ & $2.0 \pm 2.9 \mathrm{TECU}$ \\
Solar maximum summer & $-2.8 \pm 0.6 \mathrm{TECU}$ & $-2.8 \pm 0.9 \mathrm{TECU}$ & $-2.9 \pm 1.7 \mathrm{TECU}$ \\
Solar minimum equinox & $-0.6 \pm 0.5 \mathrm{TECU}$ & $-1.5 \pm 1.3 \mathrm{TECU}$ & $-2.6 \pm 2.3 \mathrm{TECU}$ \\
Solar minimum winter & $-0.6 \pm 0.5 \mathrm{TECU}$ & $-0.9 \pm 0.8 \mathrm{TECU}$ & $-0.8 \pm 1.3 \mathrm{TECU}$ \\
Solar minimum summer & $-0.6 \pm 0.3 \mathrm{TECU}$ & $-1.2 \pm 0.6 \mathrm{TECU}$ & $-2.3 \pm 1.5 \mathrm{TECU}$ \\
\hline
\end{tabular}

Averages and standard deviations of difference between the estimated vertical TEC and the corresponding vertical TEC given by the model based on the IRI-95 model over three locations for three seasons under solar maximum and minimum conditions.

They have presented vertical electron content profiles calculated by SUPIM up to the GPS altitude (about 20,200 $\mathrm{km}$ ) at noon and midnight in winter, summer and equinox. They have also shown that plasmaspheric electron content changes appreciably with season and latitude but very little with local time. These variations of plasmaspheric electron content are replicated in our model.

Ephemerides of the satellites are chosen to correspond to those of actual configurations of the GPS satellite on a particular day. Slant electron contents are calculated along all ray paths between satellites and receivers with elevation angles above $30^{\circ}$ at an interval of 30 seconds. Arbitrary offsets which represent the unknown instrumental biases inherent in GPS satellite and receiver are added to the electron contents calculated from the model. The satellite (receiver) biases which differ for each satellite (receiver) are determined to lie within -10 to 20 ( -50 to 50 ) TECU. The simulated GPS observations are reprocessed in the procedure described in Section 2 for the three seasons (equinox, winter, summer) under solar maximum and minimum conditions.

The simulation results in the case of solar maximum equinox are presented in Fig. 1. The figure shows diurnal variation of TEC over three locations. The red curves represent the vertical TEC derived from integration of the model electron density between 100 and the GPS altitude of 20,200 $\mathrm{km}$, which is input for the estimation procedure. The dotted points show the resultant TEC output from the estimation procedure within a range $1^{\circ} \times 1^{\circ}$ in longitude and latitude centered at the ground station at an interval of 30 seconds. The segments of the TEC curve relate to ray paths from individual satellites. The scatter of estimated TEC reflects imperfection in the estimation procedure. The difference between the input and output increase with decreasing latitude: the difference is less than 2 TECU at $43^{\circ} \mathrm{N}$ while it is around $5 \mathrm{TECU}$ at $33^{\circ} \mathrm{N}$. This fact can be explained in terms of the extent of the area covered by a GPS receiver. Since the GPS satellite is in an orbit with inclination of $55^{\circ}$, the area covered with a GPS receiver is wider at low latitudes than at high latitudes. In the procedure estimating absolute TEC, the homogeneity in the area covered by a GPS receiver is assumed. Thus, the discrepancy from this assumption is more severe with decreasing latitude.

The differences between the estimated vertical TEC and the corresponding vertical TEC given by the model are calculated at three locations for three seasons under solar maximum and minimum conditions. The average and standard deviation of these differences during the 24-hour period are listed in Table 1. Most averages are negative, which means underestimation of vertical TEC. This underestimation is qualitatively explained in terms of inaccuracy of the thinshell model assumption. To define the slant factor, we approximate the plasma distribution by a constant-density slab with lower and upper boundaries at 300 and $550 \mathrm{~km}$ altitudes, respectively. However, the actual plasma distribution extends toward the plasmasphere. Here, we compare the slant factors for the ionosphere and the plasmasphere. The slant factor at altitude $h$, which is defined as inverse of cosine of satellite zenith angle at a subionospheric point, is given as follows:

$$
S(\epsilon)=\frac{1}{\cos \left[\arcsin \left\{\frac{R}{R+h} \cos (\epsilon)\right\}\right]}
$$

where $\epsilon$ is the elevation angle of the line-of-sight from the receiver to the satellite, and $R$ is the radius of the Earth. At an elevation angle of $60^{\circ}\left(30^{\circ}\right), S$ is $1.14(1.73)$ and 1.02 (1.06) at $400 \mathrm{~km}$ and $10,000 \mathrm{~km}$ altitude, respectively; that is, $S$ for the plasmasphere is smaller than that for the ionosphere. The ratio between these two increases with decreasing elevation angles and reaches 0.6 at elevation angle of $30^{\circ}$. This causes overestimation of the slant factor. As a result, the equivalent vertical TEC, to which the slant TEC is converted by dividing the slant factor, is underestimated. The underestimation is largest for the solar maximum summer condition, for which the plasmaspheric electron content is highest. From Table 1, it is found that the estimated TEC is less than TEC integrated up to the GPS altitude of 20,200 $\mathrm{km}$; the difference is less than 3 TECU. The standard deviation of the difference between the two is less than 1 TECU (3 TECU) at the northern (southern) part of Japan.

As described above, the plasma distribution is approximated by a constant-density slab with lower and upper boundaries at 300 and $550 \mathrm{~km}$ altitudes, respectively. Kawamura et al. (2000) show that $F 2$ peak altitude changes from $230 \mathrm{~km}$ to $400 \mathrm{~km}$ depending on time of day, season, and solar activity over the MU radar. We have estimated change in the slant factor $S$ by changing the slab altitude. 
The slant factor at an elevation angle of $45^{\circ}$ decreases by $2 \%$ when the slab altitude increases by $200 \mathrm{~km}$. The decrease of the slant factor becomes smaller for smaller zenith angle. Therefore, we conclude that the altitude change of the $F$ layer does not cause significant errors in estimating absolute TEC.

Latitudinal variation of the plasmaspheric electron content also limits accurate determination of vertical TEC from GPS data. Lunt et al. (1999a) have showed that vertical TEC estimated on ray paths to the south of the European mid-latitude station exceeds that on ray paths to the north by an average of 0.7 TECU. Accurate estimation of vertical TEC requires to know the plasmaspheric electron content distribution.

It is concluded from the present model simulation that our TEC estimation procedure may underestimate actual TEC by less than 3 TECU. The magnitude of underestimation mainly depends on the plasmaspheric electron content. The estimated TECs scatter with the standard deviation of less than 1 TECU (3 TECU) at the northern (southern) part of Japan. It should be noted that these errors are mainly caused by the two assumptions; one is the thin-shell model assumption and the other is the homogeneity of vertical TEC in the area covered with a GPS receiver.

\subsection{Comparison with MU radar observations}

To validate this estimation method of vertical TEC over Japan, the estimated absolute GPS TECs are compared with the incoherent scatter MU radar observations. The MU radar is located at Shigaraki $\left(34.85^{\circ} \mathrm{N}, 136.10^{\circ} \mathrm{E}\right)$. Details of the MU radar system have been described by Fukao et al. (1985a, b). Altitude profiles of the electron density are obtained from power profile measurements of the incoherent echoes from the ionospheric electrons. A pulse width of $512 \mu \mathrm{s}$ is used to maximize signal-to-noise ratio. For this long-pulse measurement, the signal-to-noise ratio is larger than unity over a considerable altitude range around the $F 2$ peak altitude. The received power profiles are integrated to form hourly profiles. The number of integration is approximately 240,000 , yielding random statistical errors of the signal power less than $1 \%$ around the $F 2$ peak altitude. The received power profiles are corrected for range dependence and electron-ion temperature ratio. The temperature ratio is taken from a model based on the MU radar observations during the period from 1986 to 1997 (Otsuka et al., 1998). The resulting power profiles are normalized to the $F 2$ layer peak density using critical frequency for the $F 2$ layer, $f_{o} F 2$, obtained from an ionosonde at Kokubunji $\left(35.7^{\circ} \mathrm{N}, 139.5^{\circ} \mathrm{E}\right)$ to produce the electron density profiles. The electron density observed with the MU radar is integrated from $100 \mathrm{~km}$ to $1,000 \mathrm{~km}$ altitude to obtain vertical TEC. Since the random statistical error of the MU radar signal is less than $1 \%$, the expected error of TEC is determined mainly by the resolution of $f_{o} F 2(0.1 \mathrm{MHz}) . f_{o} F 2$ is $7-8 \mathrm{MHz}$ during daytime and 4-6 MHz during nighttime for the period of May 6-8, 1998. The error of MU radar TEC is thus $2-5 \%$.

The estimated vertical GPS TECs from the GEONET are compared to TECs derived from the MU radar observations. Figure 2 shows temporal variations of the GPS and MU radar vertical TECs over the MU radar for three consecutive days, May 6-8, 1998. The geomagnetic conditions were (a)

May 6, 1998

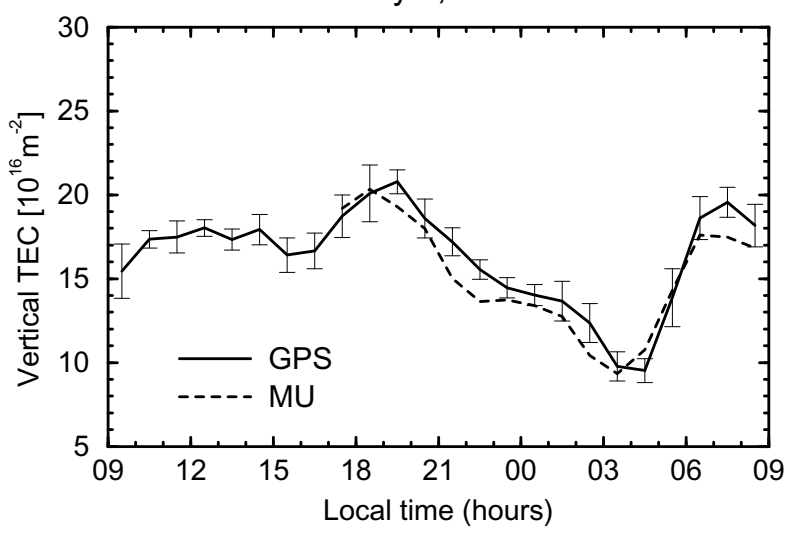

(b)

May 7, 1998

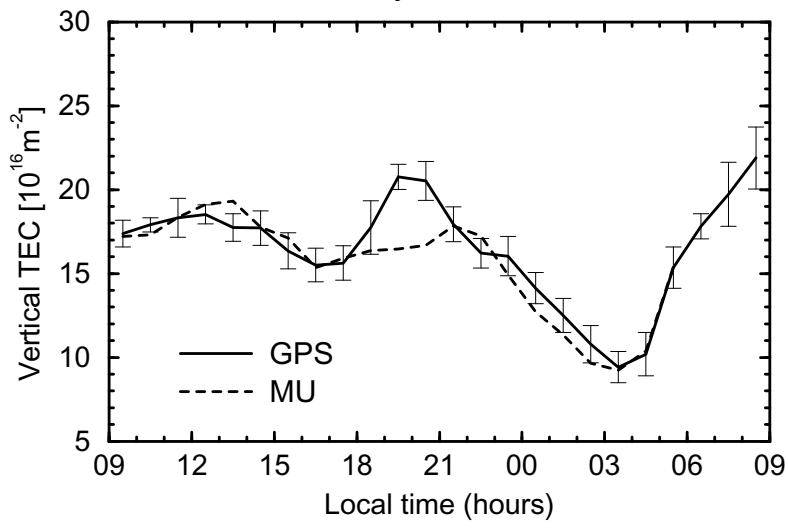

(c)

May 8, 1998

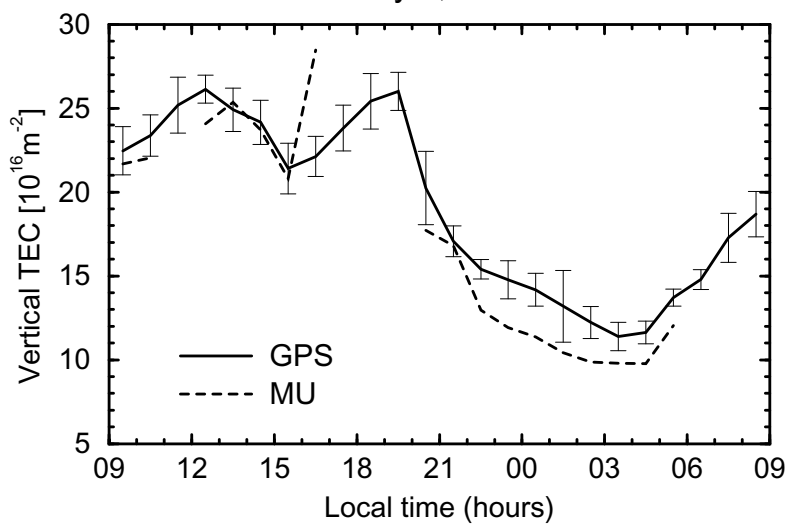

Fig. 2. Comparison of diurnal variations of TEC over Shigaraki derived from GPS GEONET and from MU radar observations on (a) May 6, (b) May 7, and (c) May 8, 1998. The GPS TEC is averaged over one hour within the area of $1^{\circ} \times 1^{\circ}$ in longitude and latitude centered at the MU radar. The error bar shows its standard deviation. The MU radar TEC is obtained by integrating electron density from 100 to $1,000 \mathrm{~km}$ altitude.

quiet on May 6, $7\left(K_{p}=1 \sim 3\right)$, and moderately disturbed on May $8\left(K_{p}=2+\sim 4+\right)$, though a geomagnetic storm occurred on May 4, 1998. Solar activity was low; 10.7-cm solar flux averaged over 81 days was 110 . The estimated GPS TECs are averaged over one hour within the area of $1^{\circ}$ in latitude and $1^{\circ}$ in longitude centered at the MU radar (standard deviations are shown by error bars) to obtain the same time and spatial resolutions as the MU radar TEC. The data gaps in the MU radar TECs are caused by interferences 
from other radio sources.

As shown in Fig. 2, both the GPS and MU radar TECs decrease from a maximum after sunset, reach a minimum just before sunrise, and then increase in the morning. It is obvious that diurnal and day-to-day variations of the GPS TEC are similar to those of the MU radar TEC, although the GPS TEC magnitude exceeds the MU radar TEC by 2 TECU on average. The difference is considered to represent the plasmaspheric TEC because the GPS altitude is about 20,200 km while the MU radar TEC is derived from the integration up to $1,000 \mathrm{~km}$ altitude. It should be noted that the TEC is underestimated by about 1-2 TECU in the solar minimum summer (Table 1). Therefore, the plasmaspheric TEC is about 3-4 TECU on average. This value is slightly larger than the plasmaspheric TEC estimated at mid-latitudes in the European and American sectors by Lunt et al. (1999b). This difference is due to the latitudinal variation of plasmaspheric electron content. According to Lunt et al. (1999b), the plasmaspheric electron content in the European sector is 2 TECU at the solar minimum while the corresponding value in the American sector is about 0.5 TECU. Balan et al. (2002) have obtained the plasmaspheric electron contents over Japan by comparing TEC obtained from the GEONET with that derived from SUPIM for different seasons at high solar activity. According to their study, the plasmaspheric contribution to the GPS TEC is up to 12 TECU at high solar activity, which changes appreciably with season and latitude and very little with the time of day.

A large difference between the two TECs occurs during 19-23 LT on May 7 (Fig. 2). In this period, southwestwardpropagating traveling ionospheric disturbances (TIDs) with a wavelength of several hundred kilometers and an amplitude of approximately 1 TECU were observed. It is noted that spatial variation (less than $1,000 \mathrm{~km}$ ) of TEC induced by TIDs may cause estimation errors of the GPS TEC to a certain degree because our method assumes that TEC is uniform within the area covered by a receiver. Ogawa et al. (2002) have studied TIDs using vertical GPS TEC data and find a coincidence of TID-related structures in TEC and 630$\mathrm{nm}$ airglow intensity. They show that fluctuation amplitudes of TEC are 0.5-2.5 TECU (relative amplitudes of a few to $20 \%$ ).

The discrepancy between the two TEC also occurs at 16 LT on May 8. The MU radar TEC increases more rapidly than the GPS TEC, and exceeds the GPS TEC by 5 TECU. This situation is physically impossible. The most possible explanation is that electron density profile observed with the MU radar is contaminated by interferences from other radio sources or coherent echoes which return from field-aligned irregularities embedded in sporadic $E$ layers through the antenna sidelobes (e.g., Yamamoto et al., 1991, 1992); the contamination causes overestimate of the MU radar TEC. Intense echoes which are expected to come from the fieldaligned irregularities are received during 17-19 LT on May 8 , so that the MU radar TEC is lost during this period.

From comparison between Fig. 2(a), (b), and (c), it is found that differences between GPS TEC and MU radar TEC tend to increase day by day. This indicates increase of the plasmaspheric electron content during this period. A geomagnetic storm occurred on May 4, 1998. Dst index reached a minimum of $-205 \mathrm{nT}$ at $15 \mathrm{LT}$ on May 4. A geomagnetic storm causes rapid contraction of the plasmapause and depletion of the flux tubes around the dusk on the first day of the storm. A gradual replenishment of the plasmaspheric ionization from the underlying ionosphere then occurs for a period of many days after the storm (Kersley et al., 1978; Kersley and Klobuchar, 1980). The period of May 6 8, 1998 shown in Fig. 2 corresponds to that of replenishment of the plasmaspheric plasma. Kersley and Klobuchar (1980) have presented storm-associated depression and replenishment of plasmaspheric electron content using ATS 6 observations, in which plasmaspheric electron content was provided by the difference between TEC measured by a group delay technique and that from Faraday rotation. A rapid depression of 1-2 TECU was observed during the evening of the first day of the storm, followed by a gradual replenishment over many days up to the next storm. Similar behavior of the plasmaspheric electron content also has been observed with the Japanese geostationary satellite ETS-II for the geomagnetic storm on February 15-16, 1978 (Ogawa et. al., 1980). The depression of plasmaspheric electron content was about 5 TECU and its continuation is 1.5 days.

\section{Application of the method}

In order to demonstrate performance of two-dimensional map of absolute TEC obtained from a GPS network (GEONET) in Japan and its usefulness to the ionospheric study, especially geomagnetic storm effect on the ionosphere, our method is applied to GPS data on a geomagnetic storm day, September 25, 1998. Figure 3 shows Dst index during the period September 23-28, 1998. A sudden storm commencement occurred at about 00 UT (09 LT) on September 25, 1998. Dst reached a minimum of $-207 \mathrm{nT}$ at 09 UT (18 LT).

Figure 4 shows two-dimensional maps of vertical TEC over Japan at each hour during 19-24 LT on September 25,1998 . The data coverage is $30^{\circ}-46^{\circ} \mathrm{N}$ in latitude and $128^{\circ}-146^{\circ} \mathrm{E}$ in longitude. The time resolution is 30 seconds and the spatial resolution is $0.15^{\circ} \times 0.15^{\circ}$ in latitude and longitude. A clear enhancement of TEC is seen during 19-

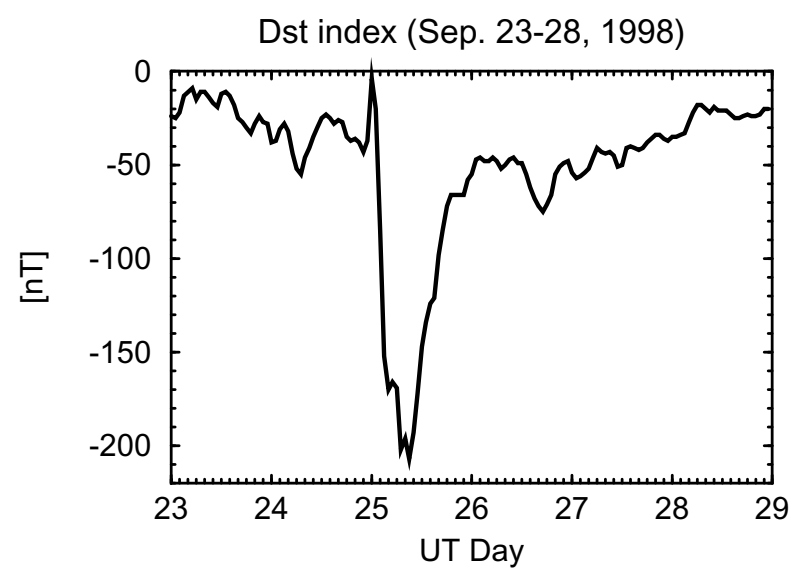

Fig. 3. Dst index during September 23-28, 1998. A sudden storm commencement occurs at about 00 UT on September 25, 1998. Dst reaches a minimum of $-207 \mathrm{nT}$ at $09 \mathrm{UT}$ on September 25 . 

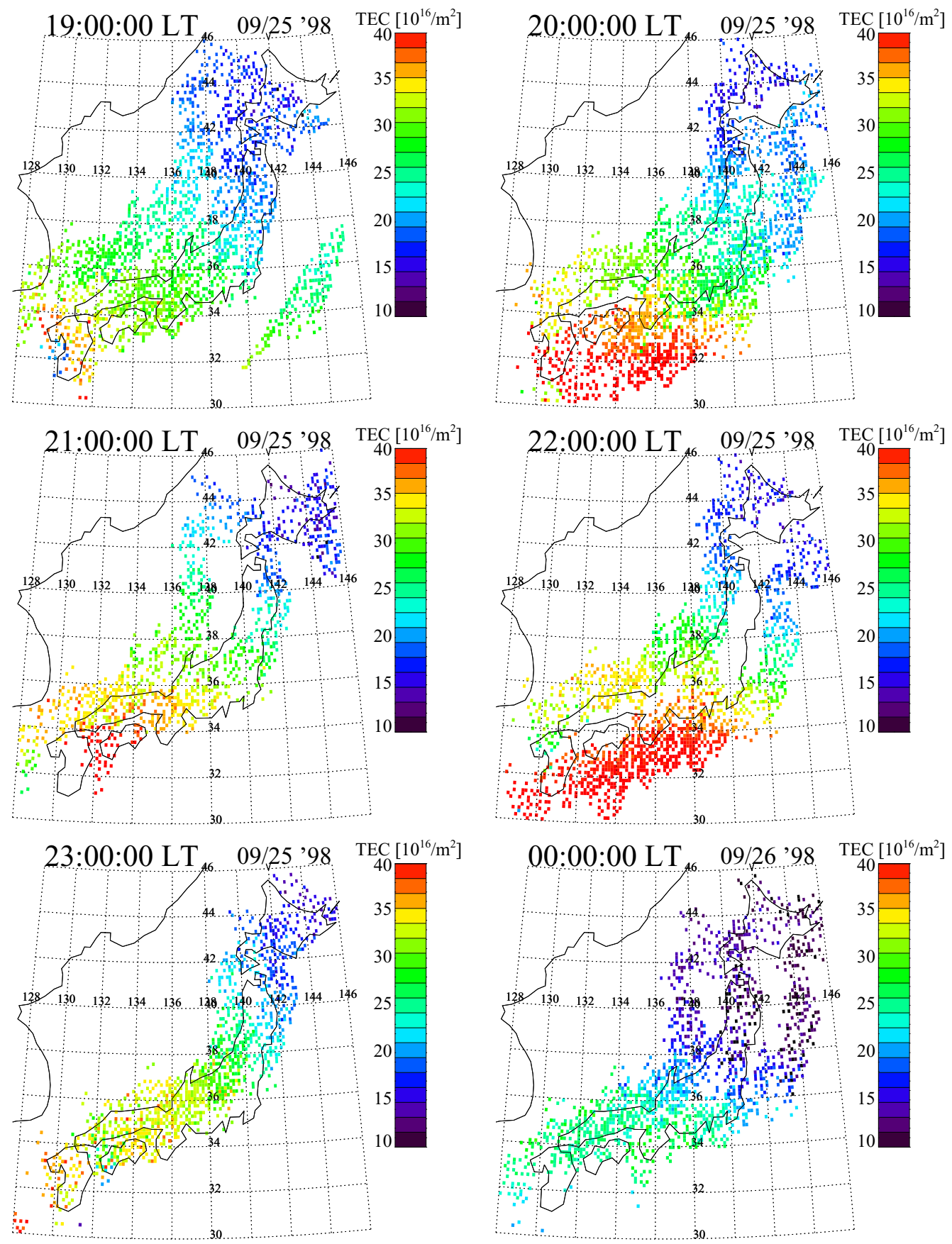

Fig. 4. Two-dimensional maps of absolute vertical TEC over Japan during 19-24 LT on September 25.

23 LT. The TEC enhancement is more intense in southern part of Japan. This is possibly associated with a poleward expansion of the equatorial anomaly. The expansion is due to storm-related enhancements of eastward electric fields at the equatorial region which cause the equatorial fountain effect.

Ogawa et al. (1980) have reported a similar phenomena, in which storm-associated enhancements of $f_{o} F 2$ and TEC over Japan were observed in the evening when horizontal component of geomagnetic field disturbances reached a minimum of $-22 \mathrm{nT}$. The enhancement of $f_{o} F 2$ was more severe and earlier in the southern part of Japan.

Buonsanto et al. (1999) have also shown a large increase in $f_{o} F 2$ observed at Arecibo during the first night of a geomagnetic storm (May 2, 1995). They have compared the results with the global maps of vertical TEC derived from GPS data and suggested that the TEC enhancement is associated with the poleward expansion of the equatorial anomaly.

The two-dimensional maps of absolute TEC with high spatial and temporal resolutions have revealed spatial structures and time evolutions of the electron density structures over Japan on the geomagnetic storm day, September 25, 1998. The characteristics of observed phenomena are consistent with the previous studies. 


\section{Summary}

We have developed a new technique to estimate absolute values of vertical TEC over Japan using GEONET data. In this technique a weighted least squares fitting is used to determine instrumental biases, assuming that hourly TEC average is uniform within an area covered by a GPS receiver. Two-dimensional maps of absolute vertical TEC are derived with a time resolution of 30 seconds and a spatial resolution of $0.15^{\circ} \times 0.15^{\circ}$ in latitude and longitude. The method does not require interpolation between TEC measurements.

Accuracy of TEC estimated from our method has been investigated using model simulations. It is found that TEC is underestimated by less than 3 TECU. The amount of underestimation mainly depends on that of the plasmaspheric electron content. The estimated TECs scatter with standard deviations of less than 1 TECU ( 3 TECU) at the northern (southern) part of Japan. These errors are mainly caused by two assumptions, that is, thin-shell model assumption and the homogeneity of vertical TEC in the area covered with a GPS receiver.

The estimated vertical GPS TECs have been compared with ionospheric electron contents calculated from electron density profiles of the MU radar. Diurnal and day-to-day variations of the GPS TEC are reasonably in agreement with those of the ionospheric electron content although the GPS TECs are larger than the ionospheric contents by 2 TECU on average. This difference can be also attributed to the plasmaspheric electron content along GPS ray path. Comparison between the GPS TEC and MU radar observations can yield storm-associated variations of the plasmaspheric electron content. Two-dimensional maps of absolute GPS TEC have reveal TEC enhancements during the main phase of the storm on September 25, 1998. The enhancement is more intense in the southern part of Japan, and is possibly caused by an expansion of the equatorial anomaly due to an enhancement of eastward electric field in the equatorial region. Continuous observations of absolute TEC over wide area with high temporal and spatial resolutions will contribute to more extensive study and monitoring of the ionosphere.

Acknowledgments. GEONET GPS data were provided by the Geographical Survey Institute of Japan. Ionograms at Kokubunji were provided by the Communications Research Laboratory, Tokyo. The MU radar is owned and operated by the Radio Science Center for Space and Atmosphere of Kyoto University. The authors would like to thank N. Balan and K. Shiokawa for their helpful comments and suggestions. This work was supported by Grand-in-Aid for Scientific Research of the Ministry of Education, Culture, Sports, Science and Technology of Japan (12554016).

\section{References}

Balan, N., Y. Otsuka, T. Tsugawa, S. Miyazaki, T. Ogawa, and K. Shiokawa, Plasmaspheric electron content in the GPS ray paths over Japan under magnetically quiet conditions at high solar activity, Earth Planets Space, 54, this issue, 71-79, 2002.

Belwitt, G., An automatic editing algorithm for GPS data, Geophys. Res. Lett., 17, 199-202, 1990.
Bilitza, D. (Ed.), International Reference Ionosphere 1990, Rep. NSSDC, 90-22, National Space Science Center, Greenbelt, MD, 1990.

Bishop, G. J., A. Mazzella, E. Holland, and S. Rao, Algorithms that use the ionosphere to control GPS errors, in Proceedings of the IEEE 1996 Position Location and Navigation Symposium (PLANS) pp. 145-152, IEEE Press, Piscataway, N. J., 1996.

Bishop, G. J., D. S. Coco, N. Lunt, C. Coker, A. J. Mazzella, and L. Kersley, Application of SCORE to extract protonospheric electron content from GPS/NNSS observations, in Proceedings of ION GPS '97, pp. 207-216, Inst. of Navig., Alexandria, Va., 1997.

Buonsanto, M. J., S. A. González, X. Pi, J. M. Ruohoniemi, M. P. Sulzer, W. E. Swartz, J. P. Thayer, and D. N. Yuan, Radar chain study of the May, 1995 storm, J. Atmos. Solar Terr. Phys., 61, 233-248, 1999.

Davies, K., Ionospheric Radio, IEE Electromagnetic Waves Series, 31, Peter Peregrinus, London, 1990.

Fukao, S., T. Sato, T. Tsuda, S. Kato, K. Wakasugi, and T. Makihira, The MU radar with an active phased array system, 1, Antenna and power amplifiers, Radio Sci., 20, 1155-1168, 1985a.

Fukao, S., T. Tsuda, T. Sato, S. Kato, K. Wakasugi, and T. Makihira, The MU radar with an active phased array system, 2, Inhouse equipment, Radio Sci., 20, 1169-1176, 1985 b.

Ho, C. M., A. J. Mannucci, U. J. Lindqwister, X. Pi, and B. T. Tsurutani, Global ionosphere perturbations monitored by the worldwide GPS network, Geophys. Res. Lett., 23, 3219-3222, 1996.

Kawamura, S., Y. Otsuka, S.-R. Zhang, S. Fukao, and W. L. Oliver, A climatology of middle and upper atmosphere radar observations of thermospheric winds, J. Geophys. Res., 105, 12,777-12,788, 2000.

Kersley, L. and J. A. Klobuchar, Storm associated protonospheric depletion and recovery, Planet. Space Sci., 28, 453-458, 1980.

Kersley, L., H. Hajeb-Hossienieh, and K. J. Edwards, Post-geomagnetic storm protonospheric replenishment, Nature, 271, 429-430, 1978.

Lunt, N., L. Kersley, G. J. Bishop, A. J. Mazzella, and G. J. Bailey, The effect of the protonosphere on the estimation of GPS total electron content: Validation using model simulations, Radio Sci., 34, 1261-1271, 1999a.

Lunt, N., L. Kersley, and G. J. Bailey, The influence of the protonosphere on GPS observations: Model simulations, Radio Sci., 34, 725-732, 1999 b.

Mannucci, A. J., B. D. Wilson, D. N. Yuan, C. H. Ho, U. J. Lindqwister, and T. F. Runge, A global mapping technique for GPS-derived ionospheric total electron content measurements, Radio Sci., 33, 565-582, 1998.

Miyazaki, S., T. Saito, M. Sasaki, Y. Hatanaka, and Y. Iimura, Expansion of GSI's nationwide GPS array, Bull. Geogr. Surv. Inst., 43, 23-34, 1997.

Ogawa, T., K. Sinno, M. Fujita, and J. Awaka, Severe disturbances of VHF and $\mathrm{GHz}$ waves from geostationary satellites during a magnetic storm, $J$. Atmos. Terr. Phys., 42, 637-644, 1980.

Ogawa, T., N. Balan, Y. Otsuka, K. Shiokawa, C. Ihara, T. Shimomai, and A. Saito, Observations and modeling of $630 \mathrm{~nm}$ airglow and total electron content associated with traveling ionospheric disturbances over Shigaraki, Japan, Earth Planets Space, 54, this issue, 45-56, 2002.

Otsuka, Y., S. Kawamura, N. Balan, S. Fukao, and G. J. Bailey, Plasma temperature variations in the ionosphere over the middle and upper atmosphere radar, J. Geophys. Res., 103, 20,705-20,713, 1998.

Sardón, E. and N. Zarraoa, Estimation of total electron content using GPS data: How stable are the differential satellite and receiver instrumental biases?, Radio Sci., 32, 1899-1910, 1997.

Sardón, E., A. Rius, and N. Zarraoa, Estimation of the transmitter and receiver differential biases and the ionospheric total electron content from Global Positioning System observations, Radio Sci., 29, 577-586, 1994

Yamamoto, M., S. Fukao, R. F. Woodman, T. Ogawa, T. Tsuda, and S. Kato, Mid-latitude $E$-region field-aligned irregularities observed with the MU radar, J. Geophys. Res., 96, 15,943-15,949, 1991.

Yamamoto, M., S. Fukao, T. Ogawa, and S. Kato, A morphological study of mid-latitude $E$-region field-aligned irregularities revealed by the MU radar, J. Atmos. Terr. Phys., 54, 769-777, 1992.

Y. Otsuka (e-mail: otsuka@stelab.nagoya-u.ac.jp), T. Ogawa, A. Saito, T. Tsugawa, S. Fukao, and S. Miyazaki 